Historic, Archive Document

Do not assume content reflects current scientific knowledge, policies, or practices. 



\begin{tabular}{|c|c|c|c|}
\hline SEEDS & $\begin{array}{l}\text { Weizht } \\
\text { Per Bu. }\end{array}$ & $\begin{array}{l}\text { Pounds } \\
\text { Per Aere }\end{array}$ & TIME OF SEEDING \\
\hline $\begin{array}{l}\text { Alfalfa, } \\
\text { broadcast. }\end{array}$ & 60 & $10-20$ & April-August \\
\hline Alsike, broadcast & 60 & $7-10$ & February-August \\
\hline Barley, drilled 8 & 48 & $72-120$ & March-May \\
\hline $\begin{array}{l}\text { Blue Grass, } \\
\text { broadcast. }\end{array}$ & 14 & $15-20$ & Mar. Apr.-Jul. Aug. \\
\hline $\begin{array}{l}\text { Buckwheat, } \\
\text { drilled 8. }\end{array}$ & 48 & $35-60$ & June-July \\
\hline $\begin{array}{l}\text { Clover, Crims } \\
\text { broadcast... }\end{array}$ & 60 & $10-15$ & July-August \\
\hline $\begin{array}{l}\text { Clover, Red, } \\
\text { broadcast.... }\end{array}$ & 60 & $8-12$ & Feb. Apr.-August \\
\hline $\begin{array}{c}\text { Clover, Mammoth } \\
\text { broadcast.............. }\end{array}$ & 60 & $8-12$ & Feb. Apr.-August \\
\hline $\begin{array}{l}\text { Clover, Sweet, } \\
\text { hulled, broadcast }\end{array}$ & 60 & $10-20$ & March-August \\
\hline $\begin{array}{l}\text { Clover, White } \\
\text { broadcast. }\end{array}$ & 60 & $6-8$ & February-August \\
\hline $\begin{array}{l}\text { Fescue, Meadow } \\
\text { broadcast }\end{array}$ & 12 & $15-20$ & Mar. Apr.-July Aug. \\
\hline Flax, drilled 8 & 56 & $28-56$ & May \\
\hline $\begin{array}{l}\text { Millet, German } \\
\text { drilled 8 }\end{array}$ & 50 & $25-50$ & May-June \\
\hline $\begin{array}{l}\text { Millet, Hun } \\
\text { drilled } 8 \ldots\end{array}$ & 48 & $25-50$ & May-July \\
\hline $\begin{array}{l}\text { Orchard Grass } \\
\text { broadcast........ }\end{array}$ & 14 & $15-25$ & Mar. Apr.-Jul. Aug. \\
\hline $\begin{array}{l}\text { Peas, Canada, } \\
\text { drilled } 8\end{array}$ & 60 & $120-180$ & March-April \\
\hline $\begin{array}{l}\text { Rape, Dwarf } \\
\text { Essex, broadcast }\end{array}$ & 50 & $5-8$ & April-July \\
\hline $\begin{array}{l}\text { Red Top, hulled, } \\
\text { broadcast....... }\end{array}$ & 14 & $10-15$ & Mar. Apr.-Jul. Aug. \\
\hline $\begin{array}{l}\text { Rye, Winter, } \\
\text { drilled } 8\end{array}$ & 56 & $85-115$ & September-Nov. \\
\hline $\begin{array}{l}\text { Soy Beans, } \\
\text { drilled } 8\end{array}$ & 60 & $60-120$ & May-June \\
\hline $\begin{array}{l}\text { Timothy, } \\
\text { broadcast. }\end{array}$ & 45 & $10-15$ & Mar. July-Sept. \\
\hline $\begin{array}{l}\text { Vetch, Spring, } \\
\text { drilled } 8\end{array}$ & & $30-60$ & April-May \\
\hline $\begin{array}{l}\text { Vetch, Winter, } \\
\text { drilled } 8\end{array}$ & 60 & $30-60$ & July-September \\
\hline $\begin{array}{l}\text { Wheat, Winter, } \\
\text { drilled } 8\end{array}$ & 60 & $75-135$ & September-Oct. \\
\hline $\begin{array}{l}\text { Wheat, Spring, } \\
\text { drilled } 8 .\end{array}$ & & $90-120$ & April \\
\hline
\end{tabular}
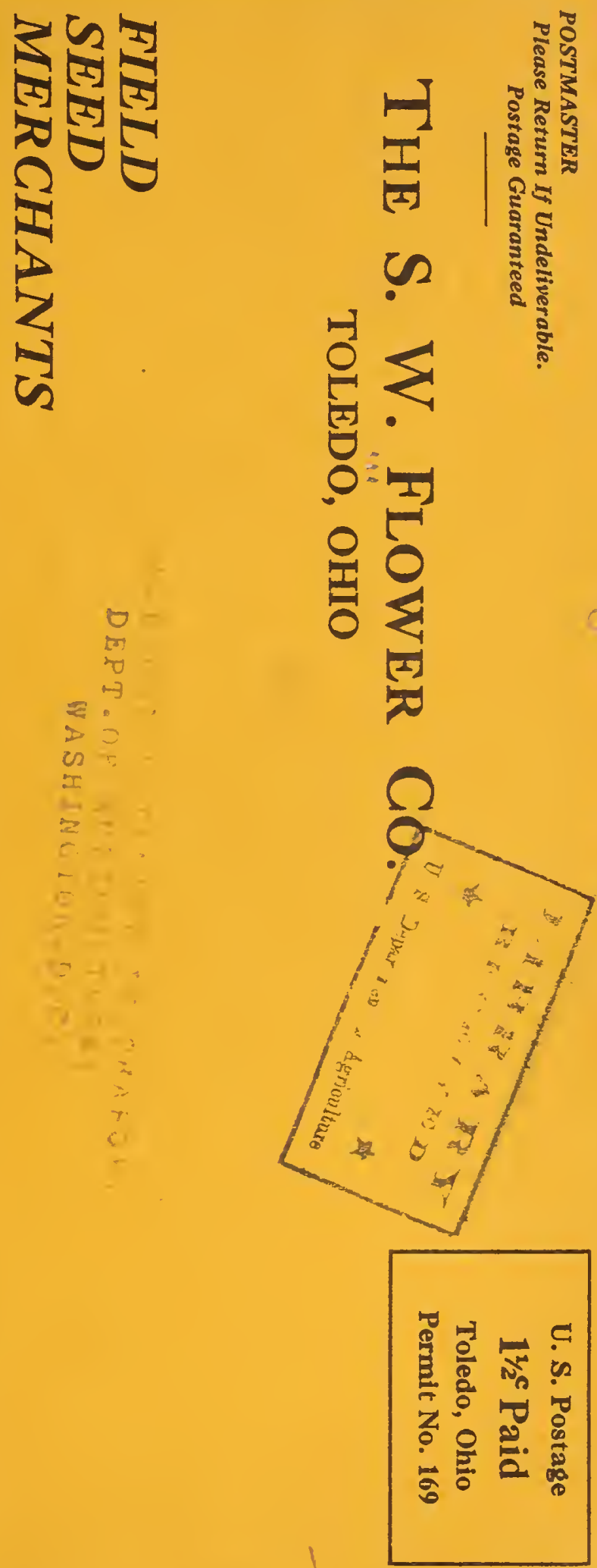



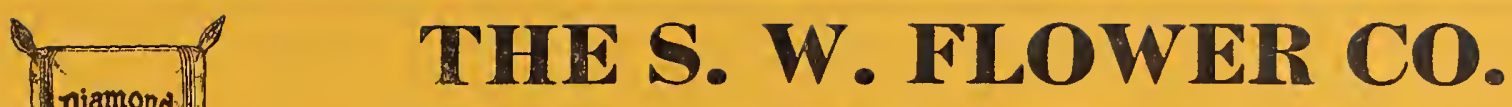

802 Lafayette St.

Next Issue, June 24 TOLEDO, OHIO

June 10, 1927
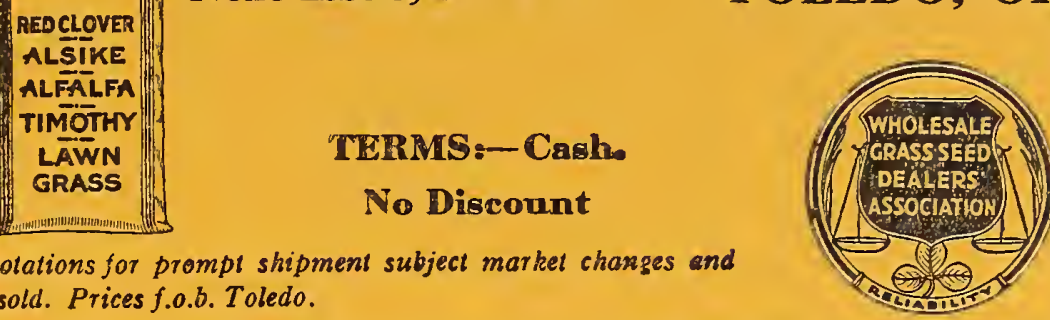

TERMS:-Cash.

No Discount

Quotations for prompt shipment subject market changes and unsold. Prices f.o.b. Toledo.

Telephone

MAin 6191

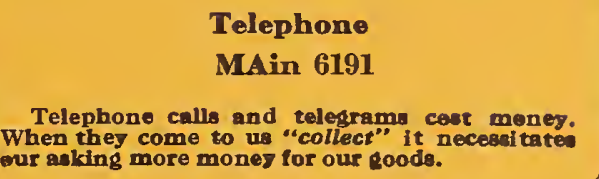
When they come to us "collect" it

Bags extra at value unless otherwise specified, not returnable Printers and manifest errors excepted.

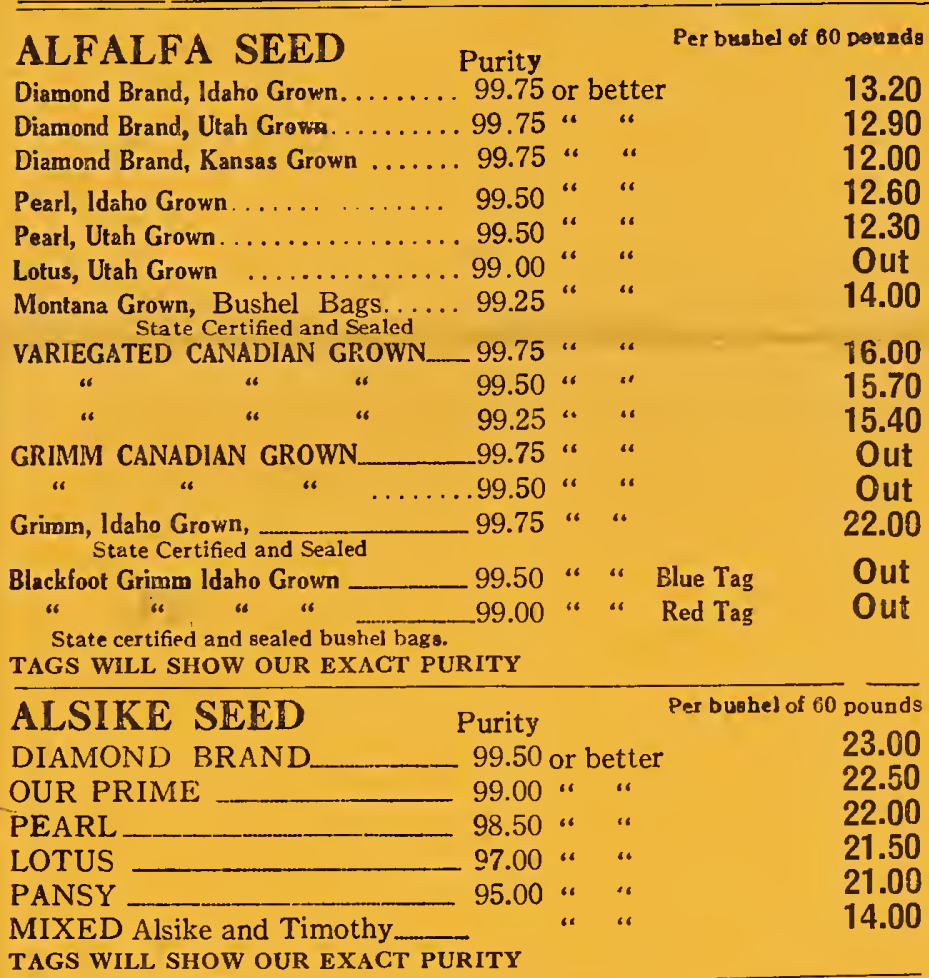

TAGS WILL SHOW OUR EXACT PURITY

\section{GLOVER SEED}

Medium - Domestic Grown

DIAMOND BRAND _._._._._._. 99.50 or better

OUR PRIME_..._... “ “

PEARL

SPECIAL-OHIO GROWN

$$
\text { IDAHO “" }
$$

Per buebel of 60 Dounds

Mammoth - Domestic Grown

OUR PRIME ..............99.50 “ “

PEARL...................... "

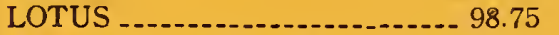

Medium-Imported-French

About $1 \%$ stained green according to

Federal Law

No. 1

No. 2 99.50 “

99.25 “" “

No. 3

98.00

.99 .50

TAGS WILL SHOW OUR EXACT PURITY

\section{SWEET CLOVER ALL SCARIFIED}

\section{White Blossom Purity}

Diamond Brand.-. Purity Per bushel of 60 ooundo

99.75 or better

8.40

8.20

Pearl

99.50 “" “

Yellow Blossom

Diamond Brand

98.65

7.50

Pearl Brand

99.75 “ “

8.40

8.20

Grundy County (Wh. Blos'm)

Diamond Brand................... 99.75 .

Pearl Brand........ “

TAGS WILL SHOW OUR EXACT PURITY

15.00

27.00

26.00

25.00

8.40

\begin{tabular}{|c|c|c|c|}
\hline TIMOTHY SEED & Purity & Per bushel & of \\
\hline DIA & -99.75 & or better & 2.95 \\
\hline OUF & 99.60 & $"$ & 2.80 \\
\hline PEA & -99.50 & " " & 2.65 \\
\hline LOT & 99.00 & " " & 2.50 \\
\hline
\end{tabular}

Out
Quality considered, you cannot beat our prices on all seeds

MIXED (Timothy and Alsike)

3.60

TAGS WILL SHOW OUR EXACT PURITY

BAGS Per 100

Cincinnati-A (new 16 oz.) $\quad 30.00$

Patched ._._. (new 1600

BuCKwheat Per $100 \mathrm{Lbs}$.

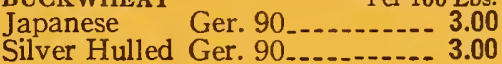

FLAX SEED

Per 100 Lbs.

\section{GRASS SEEDS}

BLUE GRASS (Gross for net sacks free)

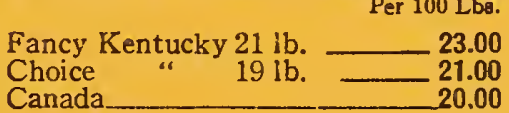

Canada “ 19 ib.

LAWN GRASS

Diamond Brand Fer $100 \mathrm{Lbs}$.

Pearl (Gross for net sacks free) $\quad 30.00$

ORCHARD GRASS (Gross for net sacs free)

26.50 Choice Per $100 \mathrm{Lbs}$.

RED TOP

Extra Fancy Punty 98.20_Per 10r) Lbs Fancy " $90.00 \square 26.00$

RYE GRASS

Per 100 L.be.

Italian 12.50

\begin{tabular}{|c|c|c|}
\hline ITTE 9 & & Per 100 \\
\hline $\begin{array}{l}\text { iamond } \\
\text { earl }\end{array}$ & & \\
\hline tus $\{96.00 \mathrm{pu}$ & tains & \\
\hline
\end{tabular}

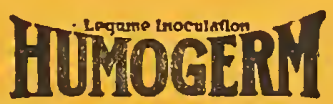

No. 1 For Medium, Mammoth, Alsike No. 2 For Alfalfa and Sweet Clover

$1 / 4 \mathrm{Bu} .24 \mathrm{c} \quad 1 \mathrm{Bu} . \ldots \quad 67 \mathrm{c}$
$1 / 2 \mathrm{Bu} . \quad 40 \mathrm{c}$

No. 9 For Soy Beans

$1 / 2$ Bu._24c 1 Bu._40c.

Postage Extra
MILLET

Per $100 \mathrm{Lb}$

Choice German Ger. 90......... 5.00

White Wonder Ger. 90_........ 4.50

Hungarian Ger. 76...........4.00

PEAS, (Canadian Field). (Grosa for net
saca free). No. 1 .....-..- Out

RAPE (Dwarf Eesez) (Gross for net wacs free

Genuine Holland Grown____ $\mathbf{7 . 0 0}$ Purity 99.75 Germination 90

SEED CLEANERS (Clipper Price

No. 1B (With Knocker)___ $\$ 28.85$ fob

No. $2 \mathrm{~B}$

Each mill equipped with 12 Screens, suitable for cleaning and grading grain, beans, corn,

POWER PULLEY included with
No. 2 B; 50 c extra with No. $1 \mathrm{~B}$

SEED TRYERS

Each $\$ 1.00$

SEWING TWINE

Per Lb.

SOY BEANS

Mammoth Yellow____ Per Bu. of 60 Lbo. Manchu _Wer. 85_ 2.50 " " 95 3.00

Wilson Blacks F.O.B. Fostoria, O.- 3.00 SUDAN GRASS

Per 100 lbs.

No. 1._._-_-_-_._-_...... 9.00

SUNFLOWER (Gross for net sacka free) Per 100 Lbs.

For Seed....................... Out

For Feed................... Out

VETCHes Per $100 \mathrm{Lba}$

Hairy or Winter Impd... P9u.40 17.50

Spring Impd............... $99.75 \quad \mathbf{7 . 5 0}$

Hairy or Winter Domes-

tic

${ }^{6}$ We will put up orders, weighing gross for net, half bushel lots or less in half bushel bags, bags extra at 15c; bushel to half bushel lots in bu= shel bags, bags extra at $25 \mathrm{c} .99$

We give no warranty, expressed or implied as to description, quality, productiveness or any other matter of any seed we sell and will not be responsible for the crop. We have an up-to-date laboratory and give tests as we find them. We think they are as near correct as possible.

We assume no responsibility whatsoever for loss or damage, for delaysin delivery or for non-delivery of seeds occasioned by fires, floods, strikes, embargoes, car shortages, or by ang other causes beyond our control. 
If you have seed to sell send samples

with lowest prices

Before buying take our qualities into consideration

Samples on request

"The Best is the Cheapest"

We sell for future shipment

If interested ask for quotations

Better Seeds

Better Crops 